\title{
In vivo antimuscarinic actions of the third generation antihistaminergic agent, desloratadine G Howell III ${ }^{\dagger 1}$, L West ${ }^{\dagger 1}$, C Jenkins ${ }^{2}$, B Lineberry ${ }^{1}$, D Yokum ${ }^{1}$ and R Rockhold*1
}

Address: ${ }^{1}$ Department of Pharmacology and Toxicology, University of Mississippi Medical Center, Jackson, MS 39216, USA and ${ }^{2}$ Tougaloo College, Tougaloo, MS, USA

Email: G Howell - ghowell@pharmacology.umsmed.edu; L West - lashondrapowell@yahoo.com; C Jenkins - colibrinecole@aol.com; B Lineberry - Larry.Lineberry@usma.edu; D Yokum - DVyokum@bsc.edu; R Rockhold* - rrockhold@pharmacology.umsmed.edu

* Corresponding author †Equal contributors

Published: 18 August 2005

BMC Pharmacology 2005, 5:13 doi:10.1186/1471-2210-5-13
Received: 06 October 2004

Accepted: 18 August 2005

This article is available from: http://www.biomedcentral.com/I47/-22/0/5/13

(C) 2005 Howell et al; licensee BioMed Central Ltd.

This is an Open Access article distributed under the terms of the Creative Commons Attribution License (http://creativecommons.org/licenses/by/2.0), which permits unrestricted use, distribution, and reproduction in any medium, provided the original work is properly cited.

\begin{abstract}
Background: Muscarinic receptor mediated adverse effects, such as sedation and xerostomia, significantly hinder the therapeutic usefulness of first generation antihistamines. Therefore, second and third generation antihistamines which effectively antagonize the $\mathrm{H}_{1}$ receptor without significant affinity for muscarinic receptors have been developed. However, both in vitro and in vivo experimentation indicates that the third generation antihistamine, desloratadine, antagonizes muscarinic receptors. To fully examine the in vivo antimuscarinic efficacy of desloratadine, two murine and two rat models were utilized. The murine models sought to determine the efficacy of desloratadine to antagonize muscarinic agonist induced salivation, lacrimation, and tremor. Desloratadine's effect on the cardiovascular system was explored in both rodent models.
\end{abstract}

Results: In the pithed rat, both desloratadine $(1.0 \mathrm{mg} / \mathrm{kg}$, i.v. $)$ and the muscarinic $M_{2}$ selective antagonist, methoctramine $(0.5 \mathrm{mg} / \mathrm{kg}$, i.v.), inhibited negative inotropic (left ventricular $\mathrm{dP} / \mathrm{dt}$ ) effects caused by oxotremorine, a nonselective muscarinic agonist $(p<0.05)$. Negative chronotropic effects caused by oxotremorine were inhibited by desloratadine, methoctramine, and the muscarinic $M_{3}$ selective antagonist, 4$\operatorname{DAMP}(1.0 \mathrm{mg} / \mathrm{kg}$, i.v.). A late positive inotropic event observed after the initial decrease was inhibited by all three test compounds with desloratadine and 4-DAMP being the most efficacious. In the conscious animal, inhibition of baroreflex-mediated bradycardia was evaluated. Unlike atropine $(0.5 \mathrm{mg} / \mathrm{kg}$, i.v.), desloratadine did not alter this bradycardia. The antimuscarinic action of desloratadine on salivation, lacrimation, and tremor was also explored. In urethane-anesthetized ( $1.5 \mathrm{~g} / \mathrm{kg}$, i.p.) male ICR mice $(25-35 \mathrm{~g})$ desloratadine $(1.0,5.0 \mathrm{mg} / \mathrm{kg})$ did not inhibit oxotremorine-induced $(0.5 \mathrm{mg} / \mathrm{kg}$, s.c.) salivation, unlike atropine $(0.5 \mathrm{mg} / \mathrm{kg})$ and 4-DAMP $(1.0 \mathrm{mg} / \mathrm{kg})$. In conscious mice, desloratadine failed to inhibit oxotremorine-induced $(0.5 \mathrm{mg} / \mathrm{kg}$, s.c.) salivation, lacrimation, and tremor. However, desloratadine did inhibit oxotremorine-induced tremor in phenylephrine pretreated animals.

Conclusion: The presented data demonstrate that the third generation antihistamine, desloratadine, does not significantly antagonize peripheral muscarinic receptors mediating salivation and lacrimation, therefore, xerostomia and dry eyes should not be observed with therapeutic use of desloratadine. Our data also indicate when administered to a patient with a compromised blood-brain barrier, desloratadine may cause sedation. Patients with compromised cardiovascular systems should be closely monitored when administered desloratadine based on our results that desloratadine has the ability to interfere with normal cardiovascular function mediated by muscarinic receptors. 


\section{Background}

Antihistaminergic drugs are commonly classified into three generations. First generation antihistamines, such as diphenhydramine, effectively block the $\mathrm{H}_{1}$ receptor subtype but their use is limited due to significant central (sedation) and peripheral (tachycardia, xerostomia) antimuscarinic side effects. Second generation antihistamines, such as loratadine, retain a high selectivity for the $\mathrm{H}_{1}$ receptor and have fewer centrally mediated side effects than the first generation compounds because second generation compounds do not readily enter the central nervous system (CNS) [1]. However, two second generation antihistamines, astemizole and terfenadine, cause prolongation of the QT interval resulting in torsades de pointes. This adverse effect prompted the removal of terfenadine from the drug market [2]. The most recent, third generation compounds, include fexofenadine and desloratadine. These compounds are active metabolites of the second generation antihistamines, terfenadine and loratadine, respectively, and generally retain or surpass the $\mathrm{H}_{1}$ receptor selectivity of their parent compounds. For instance, desloratadine displays a higher affinity for the $\mathrm{H}_{1}$ receptor than does loratadine and antagonizes the human $\mathrm{H}_{1}$ receptor in a pseudoirreversible manner $[3,4]$.

Questions remain concerning the potential for antimuscarinic adverse effects with desloratadine since both in vitro and in vivo experimentation indicates that desloratadine has the ability to block muscarinic receptors. Desloratadine demonstrated in vitro $\mathrm{IC}_{50}$ values of $48 \mathrm{nM}$ and $125 \mathrm{nM}$ against cloned human $\mathrm{M}_{1}$ and $\mathrm{M}_{3}$ muscarinic receptor subtypes, respectively [4]. In vivo muscarinic receptor blockade has been demonstrated in that desloratadine has been shown to inhibit pilocarpine induced salivation in mice and inhibit contractions of isolated rabbit and guinea pig iris smooth muscle $[5,6]$. Therefore, these data present the need to more definitively ascertain the potential antimuscarinic activity of desloratadine, in vivo. In the present study, several in vivo models were used to further assess antimuscarinic activity of desloratadine as well as the potential for penetration of the blood-brain barrier.

\section{Results}

\section{Oxotremorine-induced tremor}

Intraperitoneal injection of oxotremorine $(0.5 \mathrm{mg} / \mathrm{kg})$ induced tremor in conscious mice. The only dose of desloratadine causing inhibition of oxotremorineinduced tremor was $5.0 \mathrm{mg} / \mathrm{kg}$ (Figure 1). Desloratadine $(1.0,0.1$, and $0.01 \mathrm{mg} / \mathrm{kg})$ did not significantly inhibit generation of tremor. Unlike atropine sulfate $(0.5 \mathrm{mg} / \mathrm{kg})$, atropine methyl nitrate $(0.5 \mathrm{mg} / \mathrm{kg})$ did not inhibit tremors which confirms the central locus for oxotremorineinduced tremors. Diphenhydramine $(1.0 \mathrm{mg} / \mathrm{kg})$ significantly inhibited the generation of tremor by oxotremorine

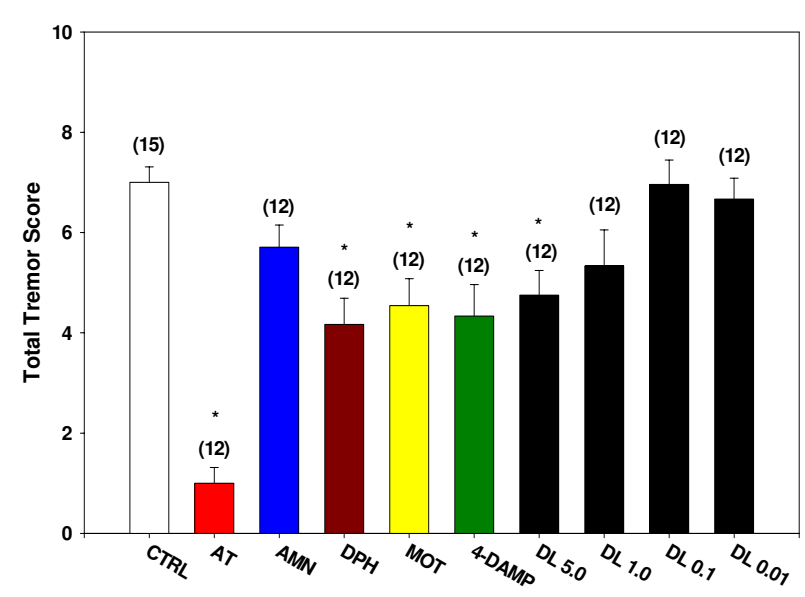

\section{Figure I}

Inhibition of oxotremorine-induced tremors. Mice were treated with a single i.p. injection of one of the test agents (atropine sulfate, AT; atropine methyl nitrate, AMN; diphenhydramine, DPH; methoctramine, MOT; I, I-dimethyl4-diphenylacetoxypiperidinium iodide, 4-DAMP; desloratadine, DL) and placed in individual shoebox cages for observation. Fifteen minutes later, each mouse received a single s.c. injection of oxotremorine sesquifumarate $(0.5 \mathrm{mg} / \mathrm{kg})$ at the nape of the neck. At 5,10 and 15 minutes following the oxotremorine injection, mice were observed for severity of tremor and for the presence of salivation and lacrimation. The sum of the scores for the three time points for tremor is presented as Total Tremor Score. Numbers in parenthesis represent the number of animals in each group and asterisk denotes statistical significance $(P<0.05)$ vs. control.

as did administration of both 4-DAMP $(1.0 \mathrm{mg} / \mathrm{kg})$ and methoctramine $(0.5 \mathrm{mg} / \mathrm{kg})$ prior to administration of oxotremorine.

\section{Oxotremorine-induced tremor with phenylephrine pretreatment}

Pretreatment with the vasopressor agent, phenylephrine $(10 \mu \mathrm{g} / \mathrm{kg}$; PE), functions to disrupt the blood-brain barrier by inducing acute hypertension $[7,8]$. Blood-brain barrier disruption resulted in significant inhibition of oxotremorine-induced tremors by desloratadine $(1.0 \mathrm{mg} /$ $\mathrm{kg}$ ) compared to the $1 \%$ DMSO vehicle as reflected by their respective total tremor scores (Figure 2). Pretreatment with PE followed by administration of desloratadine $(1.0 \mathrm{mg} / \mathrm{kg}$; i.p.) elicited an oxotremorine-induced total tremor score of $2.0 \pm 0.7$ (mean $\pm \mathrm{SD})$ whereas PE pretreatment followed by vehicle elicited an oxotremorine-induced total tremor score of $5.2 \pm 1.3($ mean $\pm S D)$. 


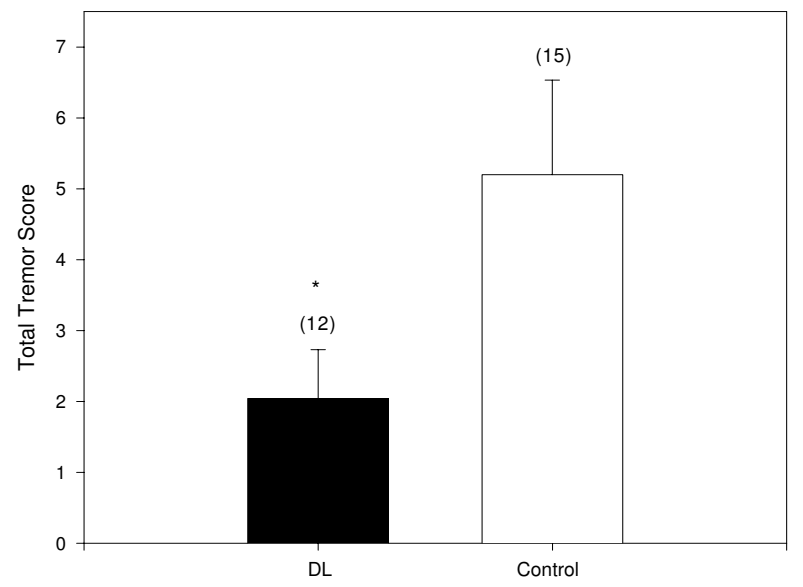

Figure 2

Inhibition of oxotremorine-induced tremors with phenylephrine pretreatment. Male ICR mice (30-35 g) were treated with a single i.p. injection of phenylephrine hydrochloride ( $10 \mathrm{mg} / \mathrm{kg}$; $10 \mu \mathrm{L} / \mathrm{g})$. Each mouse was then placed in an individual shoebox cage for observation. Five minutes after this, each mouse was given a second i.p. injection of either vehicle (control; $10 \mu \mathrm{L} / \mathrm{g}$ ) or desloratadine (free base) at a dose of $1.0 \mathrm{mg} / \mathrm{kg}(\mathrm{DL})$. Fifteen minutes later, each mouse received a single s.c. injection of oxotremorine sesquifumarate $(0.5 \mathrm{mg} / \mathrm{kg})$ at the nape of the neck. At 5,10 and 15 minutes following the oxotremorine injection, mice were observed for severity of tremor and for the presence of salivation and lacrimation. The sum of the scores for the three time points is presented as Total Tremor Score. Numbers in parenthesis represent the number of animals in each group and asterisk denotes statistical significance $(P<0.05)$ vs. control.

\section{Oxotremorine-induced salivation and lacrimation}

Administration of oxotremorine $(0.5 \mathrm{mg} / \mathrm{kg}$, i.p.) elicited salivation in conscious and urethane-anesthetized mice. In conscious mice, oxotremorine-induced salivation was not significantly inhibited by pretreatment with desloratadine $(0.01,0.1,1.0$, and $5.0 \mathrm{mg} / \mathrm{kg}$ ) (Figure 3). However, salivation was significantly inhibited by pretreatment with atropine $(0.5 \mathrm{mg} / \mathrm{kg})$, atropine methyl nitrate $(0.5$ $\mathrm{mg} / \mathrm{kg})$, and 4 -DAMP $(1.0 \mathrm{mg} / \mathrm{kg})$. Pretreatment with methoctramine $(0.5 \mathrm{mg} / \mathrm{kg})$ and diphenhydramine $(1.0$ $\mathrm{mg} / \mathrm{kg}$ ) failed to inhibit oxotremorine-induced salivation.

Inhibition of oxotremorine-induced salivation in urethane-anesthetized mice yielded results similar to those obtained in conscious male ICR mice. Pretreatment with desloratadine $(1.0 \mathrm{mg} / \mathrm{kg})$ failed to significantly inhibit

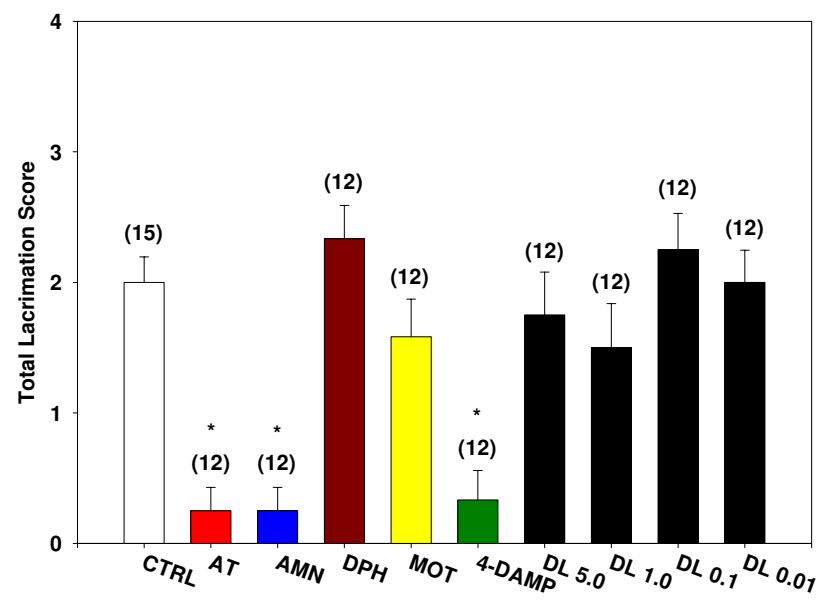

Figure 3

Inhibition of lacrimation during oxotremorine-

induced tremors. Mice were treated with a single i.p. injection of one of the test agents (atropine sulfate, AT; atropine methyl nitrate, AMN; diphenhydramine, DPH; methoctramine, MOT; I, I-dimethyl-4-diphenylacetoxypiperidinium iodide, 4-DAMP; desloratadine, DL) and placed in individual shoebox cages for observation. Fifteen minutes later, each mouse received a single s.c. injection of oxotremorine sesquifumarate $(0.5 \mathrm{mg} / \mathrm{kg})$ at the nape of the neck. At 5,10 and I 5 minutes following the oxotremorine injection, mice were observed for severity of tremor and for the presence of salivation and lacrimation. The sum of the scores for the three time points for lacrimation is presented as Total Lacrimation Score. Numbers in parenthesis represent the number of animals in each group and asterisk denotes statistical significance $(P<0.05)$ vs. control.

oxotremorine-induced salivation (Figure 4). As in the conscious animal, pretreatment with atropine $(0.5 \mathrm{mg} /$ $\mathrm{kg})$ and 4-DAMP $(1.0 \mathrm{mg} / \mathrm{kg})$ significantly inhibited oxotremorine-induced salivation. Also, administration of diphenhydramine $(1.0 \mathrm{mg} / \mathrm{kg})$ and methoctramine $(0.5$ $\mathrm{mg} / \mathrm{kg}$ ) failed to significantly inhibit saliva production.

Desloratadine $(0.01,0.1,1.0$, and $5.0 \mathrm{mg} / \mathrm{kg}$ ) pretreatment had no significant effect on oxotremorine-induced lacrimation in conscious mice (Figure 5). As with inhibition of salivation in the conscious animal, pretreatment with atropine $(0.5 \mathrm{mg} / \mathrm{kg})$ and 4 -DAMP $(1.0 \mathrm{mg} / \mathrm{kg}) \mathrm{sig}$ nificantly inhibited oxotremorine-induced lacrimation. Pretreatment with either methoctramine $(0.5 \mathrm{mg} / \mathrm{kg})$ or diphenhydramine $(1.0 \mathrm{mg} / \mathrm{kg})$ did not significantly inhibit oxotremorine-induced lacrimation. 


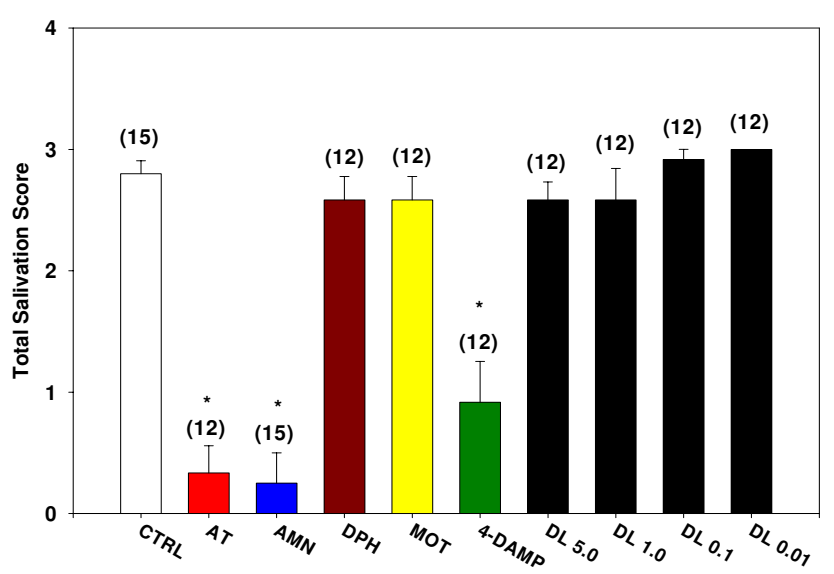

Figure 4

Inhibition of salivation during oxotremorine-induced tremors. Mice were treated with a single i.p. injection of one of the test agents (atropine sulfate, AT; atropine methyl nitrate, AMN; diphenhydramine, DPH; methoctramine, MOT; I, I-dimethyl-4-diphenylacetoxypiperidinium iodide, 4DAMP; desloratadine, DL) and placed in individual shoebox cages for observation. Fifteen minutes later, each mouse received a single s.c. injection of oxotremorine sesquifumarate $(0.5 \mathrm{mg} / \mathrm{kg})$ at the nape of the neck. At 5,10 and $15 \mathrm{~min}$ utes following the oxotremorine injection, mice were observed for severity of tremor and for the presence of salivation and lacrimation. The sum of the scores for the three time points for salivation is presented as Total Salivation Score. Numbers in parenthesis represent the number of animals in each group and asterisk denotes statistical significance $(P<0.05)$ vs. control.

\section{Oxotremorine-induced changes in left ventricular contractility}

Intravenous injections of oxotremorine (0.00125-0.02 $\mathrm{mg} / \mathrm{kg}$ ) elicited biphasic inotropic responses. The initial phase consisted of a dose-dependent decrease in $\mathrm{dP} / \mathrm{dt}$. This decrease in inotropy began approximately 30-60 seconds after the beginning of oxotremorine injection and lasted for approximately 60 seconds. Both desloratadine and methoctramine treatments effectively blocked the negative inotropic effect (Figure 6). Administration of desloratadine $(1.0 \mathrm{mg} / \mathrm{kg})$ significantly inhibited oxotremorine-induced $(0.00125,0.0025$, and $0.02 \mathrm{mg} /$ $\mathrm{kg}$ ) decreases in $\mathrm{dP} / \mathrm{dt}$ indicated by a shift in the doseresponse curve to the right. Also, administration of methoctramine $(0.5 \mathrm{mg} / \mathrm{kg})$ significantly inhibited oxotremorine-induced $(0.0025,0.01,0.02 \mathrm{mg} / \mathrm{kg})$ decreases in $\mathrm{dP} /$ $\mathrm{dt}$ indicated by a shift in the dose-response curve to the right. After administration of test agents, an additional dose of oxotremorine $(0.04 \mathrm{mg} / \mathrm{kg})$ was administered

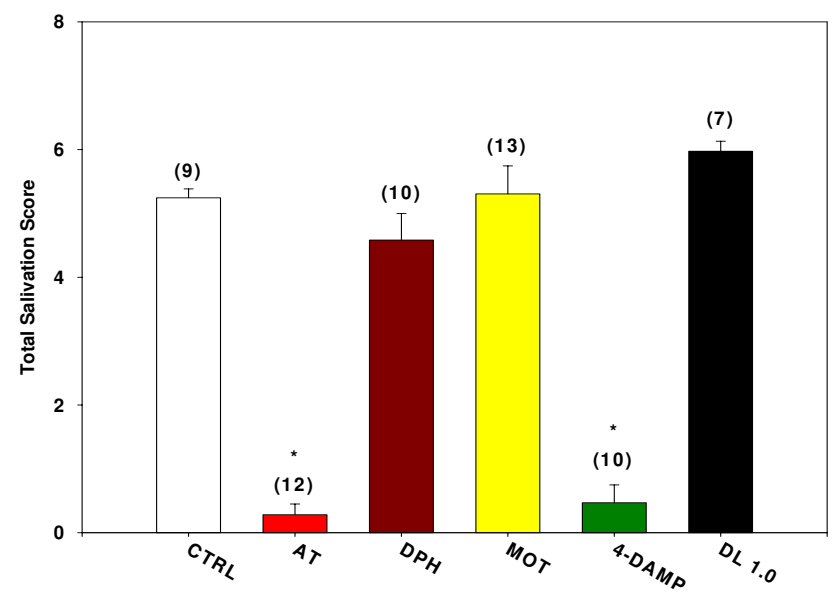

Figure 5

Inhibition of oxotremorine-induced salivation. Mice were anesthetized with urethane ( $1.5 \mathrm{~g} / \mathrm{kg}$, i.p.; I g/ml). Each mouse was then treated with a single i.p. injection of one of the test agents (atropine sulfate, AT; diphenhydramine, DPH; methoctramine, MOT; I, I-dimethyl-4-diphenylacetoxypiperidinium iodide, 4-DAMP; desloratadine, DL). Each mouse was then placed prone and head-down on a plexiglass plate inclined at $10^{\circ}$ and covered with a sheet of Whatman no. 3 MM filter paper. Fifteen minutes after administration of the test agent, a $0.5 \mathrm{mg} / \mathrm{kg}$ dose of oxotremorine was administered in a volume of $\mathrm{I} \mu \mathrm{l} / \mathrm{g}$. Every five minutes for $30 \mathrm{~min}-$ utes, each mouse was moved up the incline. Salivation production was measured immediately following the move at the end of each collection period by measurement of the circumference of the moist area of filter paper immediately beneath each mouse's mouth. The sum of the 6 collection periods is listed as Total Salivation Score. Numbers in parenthesis represent the number of animals in each group and asterisk denotes statistical significance $(P<0.05)$ vs. control.

causing percentage decreases in left ventricular (LV) contractility of $-14.2 \pm 2.7,-38.3 \pm 7.6$, and $-20.7 \pm 1.5$ for desloratadine, 4-DAMP, and methoctramine treatments, respectively (data not shown). Treatment with 4-DAMP had little antagonistic effect on the negative inotropic response to oxotremorine.

The second phase of the inotropic response to oxotremorine consisted of a dose-dependent increase in $\mathrm{dP} / \mathrm{dt}$. This increase immediately followed the initial decrease and had a duration of 2-5 minutes. Both desloratadine and 4DAMP antagonized the oxotremorine-induced positive inotropic effect (Figure 7). Administration of desloratadine $(1.0 \mathrm{mg} / \mathrm{kg})$ significantly inhibited oxotremorineinduced $(0.01$ and $0.005 \mathrm{mg} / \mathrm{kg}$ ) increases in $\mathrm{dP} / \mathrm{dt}$ indicated by a shift in the dose-response curve to the right. 

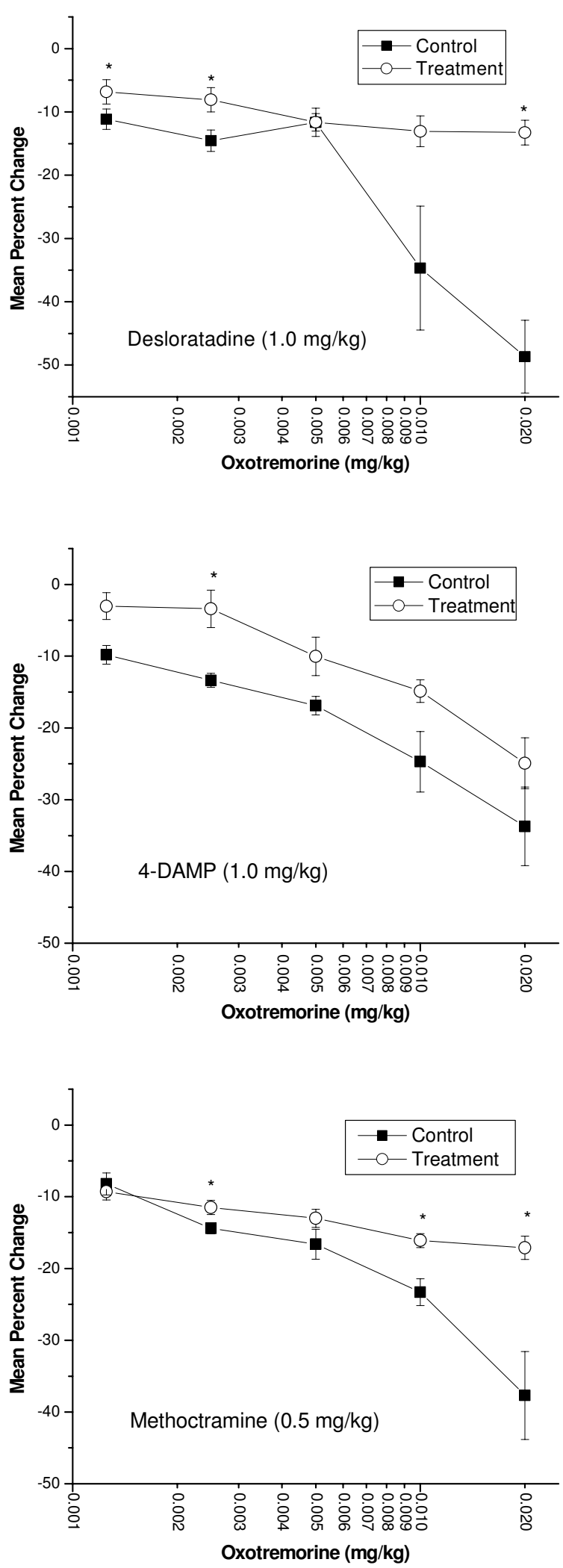

\section{Figure 6}

Effect of desloratadine (DL), 4-DAMP, or methoctramine on oxotremorine-induced decrease in left ventricular contractility in the pithed rat. Isofluraneanesthetized animals $(n=6)$ were pithed after insertion of femoral and carotid arterial catheters. The carotid catheter was advanced into the LV to enable recording of contractility, which was expressed as the change in pressure over the change in time $(\mathrm{dP} / \mathrm{dt})$. Anesthesia was then discontinued. Following administration of atenolol ( $1.0 \mathrm{mg} / \mathrm{kg}$, i.v.), oxotremorine was administered in random $(n=3)$ or ascending $(n=3)$ order of doses. The third generation antihistamine, $D L\left(1.0 \mathrm{mg} / \mathrm{kg}\right.$, i.v.), the muscarinic $M_{3}$ receptor antagonist, 4-DAMP (1.0 mg/kg, i.v.), or the muscarinic $M_{2}$ receptor antagonist, methoctramine $(0.5 \mathrm{mg} / \mathrm{kg}$, i.v. $)$, was then administered and the oxotremorine doses were repeated. No statistically significant differences were found between animals in which oxotremorine was given in random vs. ascending order of doses and both sets of data were pooled. Data are representative of the maximal percent fall in $\mathrm{dP} / \mathrm{dt}$ compared to control following the administration of each dose of oxotremorine. The highest dose tested for oxotremorine $(0.04 \mathrm{mg} / \mathrm{kg}$, i.v. $)$ could not be given prior to treatment with 4-DAMP. Statistical analysis was done using the paired t-test with $P<0.05$ denoting a statistically significant difference versus control as indicated by an asterisk.

Also, administration of 4-DAMP $(1.0 \mathrm{mg} / \mathrm{kg})$ significantly inhibited oxotremorine-induced $(0.005,0.01$, and 0.02 $\mathrm{mg} / \mathrm{kg}$ ) increases in $\mathrm{dP} / \mathrm{dt}$ indicated by a shift in the doseresponse curve to the right. In contrast to desloratadine and 4-DAMP, methoctramine treatment $(0.5 \mathrm{mg} / \mathrm{kg})$ resulted in a statistically significant $(\mathrm{P}<0.05)$ increases in $\mathrm{dP} / \mathrm{dt}$ after oxotremorine $(0.01$ and $0.02 \mathrm{mg} / \mathrm{kg})$ administration compared to control values recorded prior to methoctramine treatment. After administration of test agents, an additional dose of oxotremorine $(0.04 \mathrm{mg} / \mathrm{kg})$ was administered causing percentage increases in LV contractility of $32.2 \pm 7.1,20.5 \pm 5.4$, and $52.9 \pm 9.5$ for desloratadine, 4-DAMP, and methoctramine treatments, respectively (data not shown).

\section{Oxotremorine-induced bradycardia}

Administration of oxotremorine caused a dose-dependent decrease in heart rate. All three of the test agents antagonized this decrease as indicated by a shift in the doseresponse curve to the right (Figure 8). After administration of all three test agents, the negative chronotropic effects of oxotremorine $(0.005,0.01$, and $0.02 \mathrm{mg} / \mathrm{kg})$ were significantly inhibited. Treatment with desloratadine $(1.0 \mathrm{mg} / \mathrm{kg})$ or 4 -DAMP $(1.0 \mathrm{mg} / \mathrm{kg})$ also significantly inhibited the negative chronotropic response to oxotremorine $(0.0025 \mathrm{mg} / \mathrm{kg})$ while methoctramine $(0.5 \mathrm{mg} / \mathrm{kg})$ treatment inhibited the response to oxotremorine 

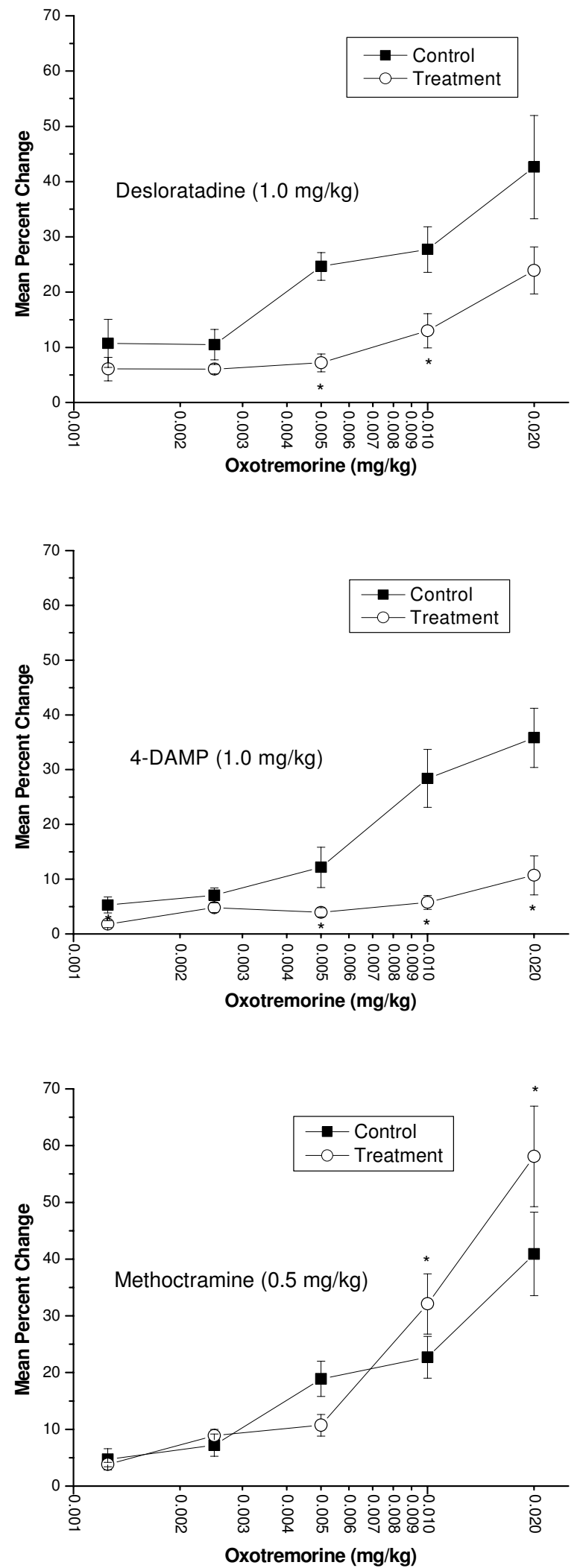

\section{Figure 7}

Effect of desloratadine (DL), 4-DAMP, or methoctramine on oxotremorine-induced increase of left ventricular contractility in the pithed rat. Isofluraneanesthetized animals $(n=6)$ were pithed after insertion of femoral and carotid arterial catheters. The carotid catheter was advanced into the LV to enable recording of contractility, which was expressed as the change in pressure over the change in time $(\mathrm{dP} / \mathrm{dt})$. Anesthesia was then discontinued. Following administration of atenolol ( $1.0 \mathrm{mg} / \mathrm{kg}$, i.v.), oxotremorine was administered in random $(n=3)$ and ascending $(n=3)$ order of doses. The third generation antihistamine, $\mathrm{DL}$ ( $1.0 \mathrm{mg} / \mathrm{kg}$, i.v.), the muscarinic $\mathrm{M}_{3}$ receptor antagonist, 4-DAMP ( $1.0 \mathrm{mg} / \mathrm{kg}$, i.v.), or the muscarinic $\mathrm{M}_{2}$ receptor antagonist, methoctramine $(0.5 \mathrm{mg} / \mathrm{kg}$, i.v. $)$ was then administered and the oxotremorine doses were repeated. No statistically significant differences were found between animals in which oxotremorine was given in random vs. ascending order of doses and both sets of data were pooled. Data are representative of the maximal percent increase in left ventricular contractility compared to control following the administration of each dose of oxotremorine. The highest dose tested for oxotremorine $(0.04 \mathrm{mg} / \mathrm{kg}$, i.v.) could not be given prior to treatment with 4-DAMP. Statistical analysis was done using the paired t-test with $\mathrm{P}<0.05$ denoting a statistically significant difference versus control as indicated by an asterisk.

$(0.00125 \mathrm{mg} / \mathrm{kg})$. After administration of test agents, an additional dose of oxotremorine $(0.04 \mathrm{mg} / \mathrm{kg})$ was administered causing percentage decreases in heart rate of $-18.8 \pm 3.5,-32.1 \pm 7$, and $-19.7 \pm 4.2$ for desloratadine, 4 DAMP, and methoctramine treatments, respectively (data not shown).

\section{Inhibition of baroreceptor reflex}

The ability of desloratadine to significantly alter the baroreceptor reflex was assessed in the conscious rat. Data were expressed as the percent change from corresponding control values of blood pressure and heart rate and subsequently analyzed by linear regression. The mean slope values were then analyzed for significant differences (data not shown). Administration of desloratadine (1.0 $\mathrm{mg} / \mathrm{kg}$ ) prior to stimulation of the baroreceptor reflex resulted in a slope value of $-0.708 \pm 0.03($ mean $\pm \mathrm{SE} ; \mathrm{n}=$ $6)$ with the corresponding control slope value of $-0.795 \pm$ $0.03(n=6)$ which was not a statistically significant difference. Unlike desloratadine, administration of atropine $(0.5 \mathrm{mg} / \mathrm{kg})$ prior to stimulation of the baroreceptor reflex resulted in a slope value of $-0.548 \pm 0.03(n=6)$ with the corresponding control slope value of $-0.670 \pm 0.02(n=6)$ which was a statistically significant difference $(P<0.05)$. 

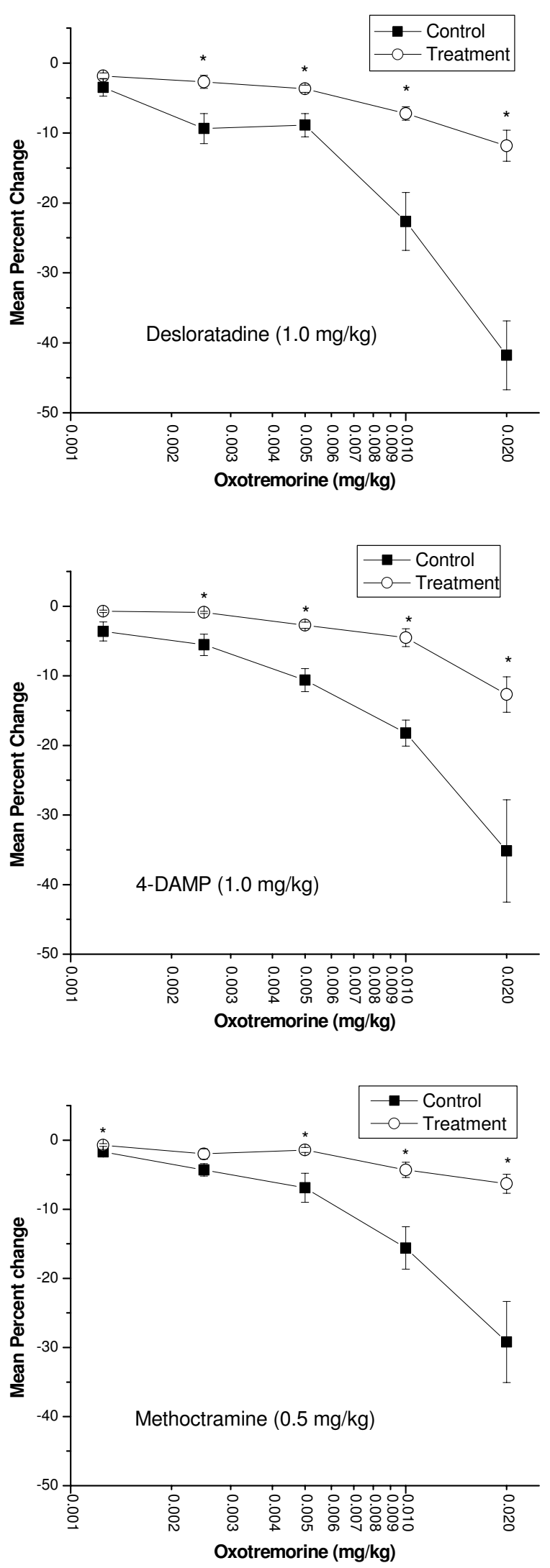

Figure 8

Effect of desloratadine (DL), 4-DAMP, or methoctramine on oxotremorine-induced decrease of heart rate $(H R)$ in the pithed rat. Isoflurane-anesthetized animals $(n=6)$ were pithed after insertion of femoral and carotid arterial catheters. The femoral catheter was inserted approximately four centimeters into the femoral artery to enable recording of heart rate. Anesthesia was then discontinued. Following administration of atenolol ( $1.0 \mathrm{mg} / \mathrm{kg}$, i.v.), oxotremorine was administered in random $(n=3)$ and ascending $(n=3)$ order of doses. The third generation antihistamine, $D L\left(1.0 \mathrm{mg} / \mathrm{kg}\right.$, i.v.), the muscarinic $M_{3}$ receptor antagonist, 4-DAMP $(1.0 \mathrm{mg} / \mathrm{kg}$, i.v. $)$, or the muscarinic $M_{2}$ receptor antagonist, methoctramine $(0.5 \mathrm{mg} / \mathrm{kg}$, i.v. $)$, was then administered and the oxotremorine doses were repeated. No statistically significant differences were found between animals in which oxotremorine was given in random vs. ascending order of doses and both sets of data were pooled. Data are representative of the maximal percent fall in heart rate compared to control following the administration of each dose of oxotremorine. The highest dose tested for oxotremorine $(0.04 \mathrm{mg} / \mathrm{kg}$, i.v. $)$ could not be given prior to treatment with 4-DAMP. Statistical analysis was done using the paired t-test with $\mathrm{P}<0.05$ denoting a statistically significant difference versus control as indicated by an asterisk.

\section{Discussion}

The focus of the present experiments was to determine the degree of antimuscarinic effects exerted by desloratadine at $\mathrm{M}_{2}$ and $\mathrm{M}_{3}$ receptors, in vivo. The non-selective muscarinic receptor agonist, oxotremorine [9], was employed as the challenge agent in the murine and rat models. Relatively selective antagonists at the $\mathrm{M}_{2}$ and $\mathrm{M}_{3}$ receptors, methoctramine $[10,11]$ and 4-DAMP $[12,13]$, respectively, were used for comparison. Our results indicate the third generation antihistaminergic agent, desloratadine, possesses a significant degree of antimuscarinic activity, primarily against cardiac $\mathrm{M}_{2}$ and $\mathrm{M}_{3}$ receptor subtypes, using in vivo whole animal preparations. However, the doses at which these activities are demonstrated exceed those normally utilized for therapeutic antihistaminergic effects. In addition, while penetration of the blood-brain barrier by desloratadine is unlikely to occur at therapeutic doses [14], evidence has been obtained suggesting penetration can be achieved and result in significant central antimuscarinic effects if the blood-brain barrier is compromised by administration of a vasopressor agent.

Oxotremorine-induced tremor, salivation, and lacrimation in the mouse have been used by others to evaluate the presence of antimuscarinic actions of drugs of interest $[13,15,16]$. The elicitation of tremor by oxotremorine is centrally mediated $[17,18]$ and blockade of this response 
gauges penetration of an antimuscarinic agent across the blood-brain barrier. Thus, blockade of oxotremorineinduced tremor is indirectly indicative of the potential for an antimuscarinic agent to exert central actions, such as sedation, following peripheral administration. In the presence of an intact blood-brain barrier, desloratadine did not exert significant blockade of oxotremorineinduced tremor, except at the highest dose tested $(5.0 \mathrm{mg} /$ $\mathrm{kg}$ ) which caused roughly $30 \%$ reduction in tremor severity. In contrast, following treatment with the vasopressor agent, phenylephrine, to open the blood-brain barrier, a previously ineffective dose of desloratadine $(1.0 \mathrm{mg} / \mathrm{kg})$ caused a $60 \%$ reduction in tremor severity. These data suggest that while desloratadine is unlikely to exert central antimuscarinic effects at therapeutic dosages $(5.0 \mathrm{mg}$ recommended dose) in normal adults, considerably greater CNS penetration may occur when the blood-brain barrier is compromised. The significance of this when desloratadine is combined with a vasopressor decongestant or when infection may compromise the blood-brain barrier $[19,20]$ remains for further study. The present results showing blockade by pretreatment with either methoctramine or 4-DAMP, indicate that oxotremorine-induced tremor is mediated by both $\mathrm{M}_{2}$ and $\mathrm{M}_{3}$ receptors in the mouse as has been previously demonstrated by others $[13,21]$.

Both oxotremorine-induced lacrimation [22] and salivation [23] have been shown to be mediated selectively through the $\mathrm{M}_{3}$ receptor subtype, a mediation confirmed by the present study. Thus, while methoctramine pretreatment had no effect on either variable, 4-DAMP pretreatment was capable of reducing both lacrimation and salivation by $60-80 \%$ below control responses. In direct contrast, desloratadine inhibited neither lacrimation nor salivation at doses as high as $5 \mathrm{mg} / \mathrm{kg}$.

The pithed, atenolol-treated rat provides a useful acute model with which to examine antimuscarinic drug action on the circulatory system in the absence of both basal and phasic sympathetic nervous system influences. The administration of oxotremorine, in this model elicits dose-dependent bradycardia, and biphasic effects on cardiac inotropy. Oxotremorine causes an initial decline in contractility, as determined by ventricular $\mathrm{dP} / \mathrm{dt}$, followed by a more prolonged positive inotropic phase. This biphasic inotropic response to a muscarinic agonist has been previously reported for acetylcholine, bethanechol, and carbachol in a variety of experimental species [24-27].

The rat heart contains multiple muscarinic receptors, including the $\mathrm{M}_{1}[28], \mathrm{M}_{2}$ [21], and $\mathrm{M}_{3}$ [29] subtypes. Of these, the $\mathrm{M}_{2}$ subtype predominates based on reverse-transcriptase polymerase chain reaction (rt-PCR) data indicating the $\mathrm{M}_{2}$ subtype constitutes more than $90 \%$ of the total muscarinic receptor mRNA, therefore, supporting its role as the major mediator of muscarinic influence over the functional state of the myocardium [30]. However, Krejci and Tucek also demonstrated the presence of mRNA for $M_{1}$ and $M_{3}$ subtypes, each constituting less than $1 \%$ and $3 \%$, respectively, of the total muscarinic receptor mRNA in the rat heart [30]. $M_{2}$ receptor agonists elicit bradycardia and a negative inotropic response through inhibition of cardiac adenylyl cyclase and/or an increase in potassium conductance via the muscarinic potassium channel [31,32]. In contrast, effects mediated through the $M_{1}$ and/ or $M_{3}$ receptors may lead to increased contractile strength, through enhanced activity of phospholipase $C$ and subsequent downstream events leading to increased intracellular free calcium availability [29,33]. Wang et al. [34] have recently reviewed the existence of multiple muscarinic receptors in the mammalian myocardium and have emphasized the presence of and physiological functions exerted by M3 receptors. A lesser body of data supports functional actions of the M1 subtype.

Desloratadine, at a dose of $1.0 \mathrm{mg} / \mathrm{kg}$, effectively antagonized bradycardia and both negative and positive inotropic responses elicited by oxotremorine. Assuming adequate selectivity between cardiac muscarinic receptor subtypes, our data suggest the ability of methoctramine to blunt oxotremorine-induced negative inotropic event and the ability of 4-DAMP to blunt oxotremorine-induced positive inotropic event to be indicative of $M_{2}$ and $M_{3}$ receptor mediation of these phenomena, respectively. In contrast, however, both methoctramine $(0.5 \mathrm{mg} / \mathrm{kg})$ and 4-DAMP (1.0 mg/kg) blunted oxotremorine-induced bradycardia. Therefore, the possibility exists that oxotremorine-induced bradycardia is mediated by both $\mathrm{M}_{2}$ and $\mathrm{M}_{3}$ receptor subtypes.

The context in which the present results are taken is worthy of discussion. Both in vitro receptor binding data [3537] and results from prior in vivo studies [5,6,37] demonstrate a considerably greater affinity of desloratadine for histaminergic than muscarinic receptors (for reviews see, $[38,39])$. Desloratadine has been found to exhibit a peak plasma concentration of approximately $28 \mathrm{ng} / \mathrm{ml}$ in healthy volunteers following a therapeutic antihistaminic dose of its parent compound, loratadine [35]. Single oral doses of desloratadine of 5, 7.5, 10, and $20 \mathrm{mg}$ yielded peak plasma concentrations of $2.18,3.03,3.80$, and 8.08 $\mathrm{ng} / \mathrm{L}$ in human volunteers [36]. In mice, desloratadine exhibits an $\mathrm{ED}_{50}$ of $0.15 \mathrm{mg} / \mathrm{kg}$ in reduction of histamine induced paw edema [37]. Cardelus et al. [5] noted local antimuscarinic effects following topical ocular administration of $1-10 \mathrm{mg} / \mathrm{ml}$ of desloratadine. However, it is unlikely that systemic concentrations of desloratadine would rise to levels approaching those in the present study following normal therapeutic dosages of deslorata- 
dine, a fact which has been emphasized by others [37] Thus, the antimuscarinic actions of desloratadine demonstrated in the present study would, most probably, be of significance only in overdose situations.

\section{Conclusion}

Our findings indicate that, at doses greater than those recommended for antihistaminergic therapy, desloratadine causes significant blockade of cardiac $\mathrm{M}_{2}$ and possibly car$\operatorname{diac} \mathrm{M}_{3}$ receptors, in vivo. This was demonstrated by significant inhibition of oxotremorine-mediated positive and negative inotropic events and bradycardia by desloratadine in the pithed rat. In contrast, desloratadine does not significantly antagonize the $\mathrm{M}_{3}$ receptor subtype responsible for salivation and lacrimation as demonstrated by the compound's inability to inhibit oxotremorine-mediated salivation and lacrimation in the conscious mouse and lacrimation in the anesthetized mouse. Also, under normal physiological conditions, desloratadine does not effectively cross the blood-brain barrier. However, upon disruption of this barrier, desloratadine has the potential for CNS penetration and muscarinic receptor blockade.

\section{Methods \\ Drugs and solutions}

Test agents included atropine sulfate, atropine methyl nitrate, diphenhydramine hydrochloride, methoctramine hydrochloride, 1,1-dimethyl-4-diphenylacetoxypiperidinium iodide (4-DAMP), and desloratadine. All were reconstituted in 1\% DMSO / PBS, aliquoted into separate vials, and stored at $-20^{\circ} \mathrm{C}$ until used. With the exception of desloratadine and 4-DAMP, all test agent concentrations were calculated using the salt weights. Atropine sulfate, atropine methyl nitrate, diphenhydramine hydrochloride, DMSO, oxotremorine sesquifumarate, atenolol, halothane, and urethane were all purchased from Sigma Chemical Co. (St. Louis, MO, USA). Other purchased agents were isoflurane (Abbott Laboratories; North Chicago, IL, USA), methoctramine hydrochloride (ICN Biochemicals, Inc.; Aurora, OH, USA), and 4-DAMP (Tocris; Ellisville, MO, USA). Desloratadine was provided by Aventis Pharmaceuticals (Bridgewater, NJ, USA).

\section{Animal experiments}

Male Sprague Dawley rats (275-325 g) and male ICR mice (25-35 g) were purchased from Harlan Sprague Dawley and housed in plastic group shoebox cages in an AAALACapproved Laboratory Animal Facility. Animals were housed under a twelve hour light-dark cycle with food and water ad libitum. Food was withheld twelve hours prior to experimentation or surgical procedures. All animal use protocols were approved by the University of Mississippi Medical Center Institutional Animal Care and Use Committee.

\section{Inhibition of oxotremorine-induced tremor, salivation, and lacrimation}

A murine model was used to test the ability of desloratadine to antagonize muscarinic actions induced by administration of the muscarinic agonist, oxotremorine [15]. On the afternoon of an experiment, each mouse was weighed and placed in a clear shoebox cage for observation 15 minutes prior to any drug administration. Test agents for this experiment were vehicle, atropine sulfate $(0.5 \mathrm{mg} /$ $\mathrm{kg})$, atropine methyl nitrate $(0.5 \mathrm{mg} / \mathrm{kg})$, diphenhydramine hydrochloride $(1.0 \mathrm{mg} / \mathrm{kg})$, methoctramine hydrochloride $(0.5 \mathrm{mg} / \mathrm{kg}), 4$-DAMP $(1.0 \mathrm{mg} /$ $\mathrm{kg}$ ), and desloratadine (5.0, 1.0, 0.1, and $0.01 \mathrm{mg} / \mathrm{kg}$ ). Each mouse was given a single intraperitoneal (i.p.) injection in a volume of $1 \mu \mathrm{l} / \mathrm{g}$ of one of the test agents. Fifteen minutes later, each mouse received a single subcutaneous (s.c.) injection of oxotremorine sesquifumarate $(0.5 \mathrm{mg} /$ $\mathrm{kg}$ ), a non-selective muscarinic agonist, at the nape of the neck. At 5, 10, and 15 minutes following oxotremorine injection, each mouse was assessed for the degree of tremor and for the presence or absence of salivation and lacrimation. A modified five-point grading scale was used to evaluate tremor: $0=$ no observable tremor; $0.5=\operatorname{limb}$ tremor observable when mouse is held by the tail with all feet off the cage bottom for 15 seconds; $1=$ intermittent tremor, with bouts lasting from 3-5 seconds; 2 = intermittent tremor, with bouts lasting more than 5 seconds; or continuous, fine tremor noticeable on tail and ears; 3 = severe, continuous, whole-body tremor. Salivation and lacrimation were separately evaluated on a two-point scale: $0=$ no observable salivation/lacrimation; $1=$ salivation/lacrimation present. All responses were assessed by each of two observers with no knowledge of the pretreatment given each mouse. The grade for each mouse reflects the sum of the three consecutive observations as either Total Tremor, Total Salivation, or Total Lacrimation Score.

\section{Inhibition of oxotremorine-induced salivation}

A second paradigm, using mice (25-35 g), anesthetized with ethyl carbamate (urethane, $1.5 \mathrm{~g} / \mathrm{kg}$, i.p., $1 \mathrm{~g} / \mathrm{ml}$ ) was used to independently evaluate putative $M_{3}$ receptor blockade inhibition of oxotremorine-induced salivation. This was modified after similar methods described for use in the rat by Lavy and Mulder [16]. All mice were denied access to food, but not to water, for 16 hours prior to anesthetization. Test agents for this experiment were vehicle, atropine sulfate $(0.5 \mathrm{mg} / \mathrm{kg})$, diphenhydramine hydrochloride $(1.0 \mathrm{mg} / \mathrm{kg})$, methoctramine hydrochloride $(0.5$ $\mathrm{mg} / \mathrm{kg}), 4$-DAMP (1.0 mg/kg; free base) and desloratadine $(1.0 \mathrm{mg} / \mathrm{kg}$; free base). A single i.p. injection of one of the test agents or vehicle was administered in a volume of 1.0 $\mu \mathrm{l} / \mathrm{g}$ five minutes following injection of urethane. Each mouse was then placed prone and head-down on a plexiglass plate inclined at $10^{\circ}$ and covered with a sheet of Whatman no. $3 \mathrm{MM}$ filter paper. Fifteen minutes after test 
agent administration, a $0.5 \mathrm{mg} / \mathrm{kg}$ (i.p.) dose of oxotremorine was given in a volume of $1.0 \mu \mathrm{l} / \mathrm{g}$ body weight. Each mouse was moved up the incline every five minutes for thirty minutes. Saliva production was quantitated at the end of each five-minute collection period by measurement of the circumference of the moist area of filter paper immediately beneath each mouse's mouth. The sum of values from the six collection periods was recorded as Total Salivation Score (TSS).

\section{Inhibition of oxotremorine-induced tremor with phenylephrine pretreatment}

Mice (30-35 g) were treated with a single i.p. injection of phenylephrine hydrochloride $(10 \mu \mathrm{g} / \mathrm{kg} ; 10 \mu \mathrm{L} / \mathrm{g})$ to elevate systemic blood pressure and open the blood-brain barrier $[7,8]$. Each mouse was then placed in an individual shoebox cage for observation. Five minutes after this, each mouse was given a second i.p. injection of either vehicle or desloratadine (free base) at a dose of $1.0 \mathrm{mg} / \mathrm{kg}$. Fifteen minutes later, each mouse received a single s.c. injection of oxotremorine $(0.5 \mathrm{mg} / \mathrm{kg})$ at the nape of the neck. At 5 , 10, and 15 minutes post oxotremorine injection, mice were observed for severity of tremor. The sum of the scores for the three time points is presented as Total Tremor Score.

\section{Inhibition of oxotremorine-induced changes in cardiac function}

The influence of oxotremorine over cardiac function in a pithed rat model was employed to evaluate muscarinic receptor antagonistic properties of desloratadine [15]. Male rats (275-325 g) were acutely anesthetized with 2$4 \%$ isoflurane in medical grade oxygen. Polyethylene arterial (PE-50) and venous (PE-10) catheters and a tracheal cannula (PE-240) were surgically implanted to permit monitoring of arterial blood pressure and chronotropy, i.v. drug administration, and maintenance of respiration by means of a Harvard rodent respirator, respectively. A catheter (PE-50) was passed via the right carotid artery into the left cardiac ventricle for measurement of left ventricular $\mathrm{dP} / \mathrm{dt}$ as an index of inotropy. Responses were obtained using either a Grass Model 7P20G differentiator and recorded on a Grass Model 7D polygraph (Grass Instrument Co.; Quincy, MASS, USA) or with PowerLab/16 SP data acquisition system using Chart for Windows v4.0 recording software (ADInstruments; Colorado Springs, CO, USA). Each rat was then pithed by insertion of a blunt stainless steel rod, 2 millimeters in diameter, through the orbit of the eye and passed through the brain and spinal column, thus destroying the central nervous system (CNS) from forebrain to the terminus of the spinal cord. Atenolol $(10 \mathrm{mg} / \mathrm{kg}, 1.0 \mathrm{ml} / \mathrm{kg}$, i.v. $)$ was administered to obviate peripheral catecholamineinduced increases in cardiac function. After a 15 minute stabilization period, doses of oxotremorine $(0.00125$,
$0.0025,0.005,0.01,0.02,0.1 \mathrm{mg} / \mathrm{kg}$ ), were administered randomly and flushed with $0.1 \mathrm{ml}$ of heparinized $0.9 \%$ saline. Subsequently, a single i.v. injection of one of three test agents was administered over a two minute period. The test agents were desloratadine $(1.0 \mathrm{mg} / \mathrm{kg}, 1.0 \mathrm{ml} / \mathrm{kg}$, i.v.), the selective $M_{3}$ muscarinic receptor antagonist, 4DAMP $(1.0 \mathrm{mg} / \mathrm{kg}, 1.0 \mathrm{ml} / \mathrm{kg}$, i.v. $)$, or the selective $M_{2}$ muscarinic receptor antagonist, methoctramine $(0.5 \mathrm{mg} /$ $\mathrm{kg}, 1.0 \mathrm{ml} / \mathrm{kg}$, i.v.). All doses of oxotremorine were then repeated in the order they were given prior to administration of the test compound. Maximal changes in chronotropy and inotropy were measured with each injection. Values are expressed as percent of the control value taken immediately before injection of each dose of oxotremorine.

\section{Inhibition of baroreceptor reflex}

Rats (300-325 g) were used to determine the ability of desloratadine to block the vagally-mediated bradycardic component of the baroreceptor reflex. Catheters were inserted into the femoral artery (PE-50) and femoral vein (PE-10) and exteriorized between the animal's shoulders. Animals were allowed to recover for a minimum of three days. The experiment lasted two days per animal. Before the baroreceptor reflex of each animal was measured, the animal was allowed an acclimation period. The first day consisted of control baroreceptor reflex measurement. On the second day, either desloratadine $(1.0 \mathrm{mg} / \mathrm{kg}$, i.v.) or atropine $(0.5 \mathrm{mg} / \mathrm{kg}$, i.v.) was given prior to baroreceptor challenge. After an acclimation period, baroreceptor reflex measurement was repeated. The baroreceptor reflex was initiated by increasing doses of both phenylephrine and sodium nitroprusside to increase or decrease blood pressure, respectively. Dosing was discontinued when a maximal change of $50 \mathrm{mmHg}$ was achieved. Blood pressure was recorded with Powerlab Data Acquisition system via a transducer attached to the arterial line. The linear regression feature in Origin 6.0 (OriginLab Corp.; Northhampton, MA) analyzed data and the slopes compared with SigmaStat 2.0 (Jandel Scientific Software; San Rafael, CA).

\section{Statistical analysis}

Data obtained from the murine models of oxotremorineinduced salivation, lacrimation, and tremor were analyzed using a one-way repeated measures ANOVA with a Dunn's post hoc test. Data obtained from animals pretreated with either phenylephrine or vehicle was analyzed via the paired t-test. Inhibition of oxotremorine-induced alterations in cardiac function before and after either desloratadine, methoctramine, or 4-DAMP administration was analyzed via the paired t-test with changes in cardiac function at each dose of oxotremorine being compared. In all statistical comparisons, $\mathrm{P} \leq 0.05$ was deemed statistically significant. 


\section{Authors' contributions}

G. Howell performed all of the in vivo cardiovascular testing, drug preparation, data analysis on cardiovascular results, and drafted the manuscript. L. West performed in vivo salivation, lacrimation, and tremor testing, drug preparation, and data analysis on salivation and lacrimation results. C. Jenkins, B. Lineberry, and D. Yokum assisted L. West with oxotremorine-induced tremor experimentation on conscious mice. R. Rockhold is the corresponding author and principal investigator.

\section{Acknowledgements}

This work was supported by a grant from Aventis Pharmaceuticals and in part by an award from the Howard Hughes Medical Institute.

\section{References}

I. Kay GG, Harris AG: Loratadine: a non-sedating antihistamine. Review of its effects on cognition, psychomotor perform ance, mood and sedation. Clin Exp Allergy 1999, 29 Suppl 3:147-150.

2. DuBuske LM: Second-generation antihistamines: the risk of ventricular arrhythmias. Clin Ther 1999, 21:28I-295.

3. Anthes JC, Gilchrest H, Richard C, Eckel S, Hesk D, West REJ, Williams SM, Greenfeder S, Billah M, Kreutner W, Egan RE: Biochemical characterization of desloratadine, a potent antagonist of the human histamine $\mathbf{H}(\mathrm{I})$ receptor. Eur J Pharmacol 2002, 449:229-237.

4. Handley DA, McCullough JR, Fang Y, Wright SE, Smith ER: Descarboethoxyloratadine, a metabolite of loratadine, is a superior antihistamine. Ann Allergy Asthma Immunol 1997, 78:PI64.

5. Cardelus I, Puig J, Bou J, Jauregui J, Llenas J, Fernandez AG, Palacios JM: Xerostomia and mydriasis, two muscarinic peripheral side effects associated with descarboethoxyloratadine, the main metabolite of loratadine. Br J Pharmacol 1998, I 23:267P.

6. Cardelus I, Anton F, Beleta J, Palacios JM: Anticholinergic effects of desloratadine, the major metabolite of loratadine, in rabbit and guinea-pig iris smooth muscle. Eur J Pharmacol 1999, 374:249-254.

7. Mayhan WG: Disruption of blood-brain barrier during acute hypertension in adult and aged rats. Am J Physiol 1990, 258:HI735-HI738.

8. Kramer JM, Aragones A, Waldrop TG: Reflex cardiovascular responses originating in exercising muscles of mice. J Appl Physiol 2001, 90:579-585.

9. Ringdahl B, Jenden DJ: Pharmacological properties of oxotremorine and its analogs. Life Sci 1983, 32:240 -24I3.

10. Giraldo E, Micheletti R, Montagna E, Giachetti A, Vigano MA, Ladinsky $H$, Melchiorre $C$ : Binding and functional characterization of the cardioselective muscarinic antagonist methoctramine. J Pharmacol Exp Ther 1988, 244: 1016-1020.

11. Wess J, Angeli P, Melchiorre C, Moser U, Mutschler E, Lambrecht G Methoctramine selectively blocks cardiac muscarinic M2 receptors in vivo. Naunyn Schmiedebergs Arch Pharmacol 1988, 338:246-249.

12. Moriya H, Takagi Y, Nakanishi T, Hayashi M, Tani T, Hirotsu I: Affinity profiles of various muscarinic antagonists for cloned human muscarinic acetylcholine receptor (mAChR) subtypes and $\mathrm{mAChRs}$ in rat heart and submandibular gland. Life Sci 1999, 64:235I-2358.

13. Sanchez C, Lembol HL: The involvement of muscarinic receptor subtypes in the mediation of hypothermia, tremor, and salivation in male mice. Pharmacol Toxicol 1994, 74:35-39.

14. Clissold SP, Sorkin EM, Goa KL: Loratadine. A preliminary review of its pharmacodynamic properties and therapeutic efficacy. Drugs 1989, 37:42-57.

15. Watanabe T, Kakefuda A, Tanaka A, Takizawa K, Hirano S, Shibata H, Yamagiwa Y, Yanagisawa I: Synthesis and biological evaluation of phenylacetyl derivatives having low central nervous system permeability as potent and selective $M 2$ muscarinic receptor antagonists. Chem Pharm Bull (Tokyo) 1998, 46:53-68.
16. Lavy UI, Mulder D: Salivary inhibition in mice and rabbits by a number of anticholinergics. A methodological investigation. Arch Int Pharmacodyn Ther 1969, 178:437-445.

17. Espinola EB, Oliveira MG, Carlini EA: Differences in central and peripheral responses to oxotremorine in young and aged rats. Pharmacol Biochem Behav 1999, 62:419-423.

18. Sanchez C, Meier E: Central and peripheral mediation of hypothermia, tremor and salivation induced by muscarinic agonists in mice. Pharmacol Toxicol 1993, 72:262-267.

19. Boje KM: Inhibition of nitric oxide synthase attenuates bloodbrain barrier disruption during experimental meningitis. Brain Res 1996, 720:75-83.

20. Mayhan WG: Effect of lipopolysaccharide on the permeability and reactivity of the cerebral microcirculation: role of inducible nitric oxide synthase. Brain Res 1998, 792:353-357.

2I. Bymaster FP, Carter PA, Zhang L, Falcone JF, Stengel PW, Cohen ML, Shannon HE, Gomeza J, Wess J, Felder CC: Investigations into the physiological role of muscarinic M2 and M4 muscarinic and M4 receptor subtypes using receptor knockout mice. Life Sci 200I, 68:2473-2479.

22. Nakamura M, Tada Y, Akaishi T, Nakata K: M3 muscarinic receptor mediates regulation of protein secretion in rabbit lacrimal gland. Curr Eye Res 1997, 16:6/4-619.

23. Schiavone A, Brambilla A: Muscarinic M3 receptors mediate secretion from sweat glands in the rat. Pharmacol Res 1991, 23:233-239.

24. Du XY, Schoemaker RG, Bos E, Saxena PR: Characterization of the positive and negative inotropic effects of acetylcholine in the human myocardium. Eur J Pharmacol 1995, 284: I I9-127.

25. Eglen RM, Montgomery WW, Whiting RL: Negative and positive inotropic responses to muscarinic agonists in guinea pig and rat atria in vitro. J Pharmacol Exp Ther 1988, 247:911-917.

26. Nishimaru K, Tanaka $Y$, Tanaka H, Shigenobu K: Positive and negative inotropic effects of muscarinic receptor stimulation in mouse left atria. Life Sci 2000, 66:607-6I5.

27. Yang JM, Chung KT, Yang SN: Muscarinic activation causes biphasic inotropic response and decreases cellular $\mathbf{N a}+$ activity in canine cardiac Purkinje fibers. J Biomed Sci 1999, 6:176-182.

28. Sharma VK, Colecraft HM, Wang DX, Levey Al, Grigorenko EV, Yeh $\mathrm{HH}$, Sheu SS: Molecular and functional identification of $\mathbf{m ~ I}$ muscarinic acetylcholine receptors in rat ventricular myocytes. Circ Res 1996, 79:86-93.

29. Ponicke K, Heinroth-Hoffmann I, Brodde OE: Demonstration of functional M3-muscarinic receptors in ventricular cardiomyocytes of adult rats. BrJ Pharmacol 2003, I38:156-160.

30. Krejci A, Tucek S: Quantitation of mRNAs for $M(I)$ to $M(5)$ subtypes of muscarinic receptors in rat heart and brain cortex. Mol Pharmacol 2002, 61: 1267-I272.

31. Dhein S, van Koppen CJ, Brodde OE: Muscarinic receptors in the mammalian heart. Pharmacol Res 200I, 44: $|6|-\mid 82$.

32. McMorn SO, Harrison SM, Zang WJ, Yu XJ, Boyett MR: A direct negative inotropic effect of acetylcholine on rat ventricular myocytes. Am J Physiol 1993, 265:HI 393-HI400.

33. Gallo MP, Alloatti G, Eva C, Oberto A, Levi RC: MI muscarinic receptors increase calcium current and phosphoinositide turnover in guinea-pig ventricular cardiocytes. J Physiol 1993, 47I:4I-60.

34. Wang Z, Shi $\mathrm{H}$, Wang $\mathrm{H}$ : Functional $\mathbf{M} 3$ muscarinic acetylcholine receptors in mammalian hearts. $\mathrm{Br} J$ Pharmacol 2004, 142:395-408.

35. Haria M, Fitton A, Peters DH: Loratadine. A reappraisal of its pharmacological properties and therapeutic use in allergic disorders. Drugs 1994, 48:617-637.

36. Gupta S, Banfield C, Affrime M, Marco A, Cayen M, Herron J, Padhi $D$ : Desloratadine demonstrates dose proportionality in healthy adults after single doses. Clin Pharmacokinet 2002, 4I Suppl I:I-6.

37. Kreutner W, Hey JA, Anthes J, Barnett A, Young S, Tozzi S: Preclinical pharmacology of desloratadine, a selective and nonsedating histamine $\mathrm{HI}$ receptor antagonist. Ist communication: receptor selectivity, antihistaminic activity, and antiallergenic effects. Arzneimittelforschung 2000, 50:345-352.

38. Geha RS, Meltzer EO: Desloratadine: A new, nonsedating, oral antihistamine. J Allergy Clin Immunol 200I, 107:75 I-762. 
39. Henz BM: The pharmacologic profile of desloratadine: a review. Allergy 200I, 56 Suppl 65:7-13.

Publish with Biomed Central and every scientist can read your work free of charge

"BioMed Central will be the most significant development for disseminating the results of biomedical research in our lifetime. " Sir Paul Nurse, Cancer Research UK

Your research papers will be:

- available free of charge to the entire biomedical community

- peer reviewed and published immediately upon acceptance

- cited in PubMed and archived on PubMed Central

- yours - you keep the copyright

Submit your manuscript here:

http://www.biomedcentral.com/info/publishing_adv.asp
BiolMedcentral 